\title{
ON \\ Utilidade do Teste de Vazamento de Bile Durante Hepatectomias
}

\section{Autores}

Hanna Vasconcelos ${ }^{1}$; Luiza Basilioo ${ }^{1}$; Rafaela Capelli ${ }^{1}$; Renato Cano ${ }^{1}$; Marcelo D'Oliveira ${ }^{1}$; Klaus Steinbruck ${ }^{2}$; Reinaldo Fernandes²; Marcelo Enne ${ }^{1}$; Eduardo Viana ${ }^{1}$;

1. Hospital Federal de Ipanema

2. Hospital Federal de Bonsucesso

Palavras-chave: fístula biliar, hepatectomia

\section{Autor correspondente:}

Hanna Vasconcelos

Rua da Matriz 70/302; (21) 97927-7227

hannavasconcelos@gmail.com

\section{RESUMO}

\section{Introduçáo}

A ressecção hepática se tornou mais segura nos últimos anos, com taxas de mortalidade menores do que $5 \%$ em grandes centros. Porém, a incidência da fistula biliar não mudou, variando de 4\%-15\%. O presente estudo tem como objetivo avaliar se o uso sistemático do teste de biliostasia durante ressecçooes hepáticas reduz a incidência de fístula biliar no pós operatório.

\section{Métodos}

De janeiro 2016 a dezembro 2018 no Hospital Federal de Ipanema foram realizadas
103 hepatectomias. Foram incluídos no estudo 85 pacientes e excluídos 19 pacientes submetidos a cirurgia videolaparoscópica, derivaçáo biliodigestiva e com dados incompletos. Os pacientes foram divididos em dois grupos: $\mathrm{A}$ - não foi realizado o teste da biliostasia no intra operatório; B - foi realizado o teste ao final da transecção hepática, após realizar colecistectomia. O ducto cístico é cateterizado e injetado $10 \mathrm{ml}$ de soro fisiologico $0,9 \%$, seguido de $10 \mathrm{ml}$ de ar duas vezes, observando se há pontos de vazamento de bile na superfície cruenta do fígado. Esses pontos de vazamento são identificados e suturados e o teste é repetido por mais duas vezes. Em todos os casos realizou-se drenagem da cavidade abdominal. Fístula biliar foi definida de acordo com os critérios propostos pelo Grupo Internacional de Estudo em Cirurgia Hepática, assim como o grau de serveridade da fístula.

\section{Resultados}

Dos 84 pacientes analisados, 46 eram mulheres e 38 homens. Quarenta pacientes $(47,61 \%)$ foram submetidos a hepatectomia sem teste da biliostasia e alocados no grupo A e 44 pacientes foram submetidos a hepa- 
tectomia com teste da biliostasia (52,39\%) e alocados no grupo B. Dos pacientes do grupo A, 5 (12,5\%) evoluíram com fístula biliar no pós operatório. Destas, uma foi classificada como grau A, 3 como grau B e 1 como grau C. Dos pacientes do grupo B, 2 $(4,54 \%)$ evoluíram com fístula biliar no pós operatório. Ambos evoluíram com fistula grau $\mathrm{B}$, porém apenas em um paciente $\mathrm{o}$ teste evidenciou vazamento de bile.

\section{Conclusáo}

A fístula biliar é uma complicação comum após ressecçóes hepáticas e afeta a qualidade de vida no pós operatório. $\mathrm{O}$ estudo mostrou que o grupo submetido ao teste de biliostasia tiveram menor incidência de fístula biliar no pós operatório $(4,45 \%)$ em relação ao não submetido ao teste $(12,5 \%)$. Isto sugere que o teste da biliostasia é um método seguro e eficaz na redução da incidência da fístula biliar no pós operatório.

\section{INTRODUÇÃO}

A ressecção hepática é o único tratamento potencialmente curativo para tumores hepáticos ${ }^{1-3}$. Durante as últimas duas décadas, a ressecção hepática se tornou mais segura, com as taxas de mortalidade menores do que $5 \%$ em grandes centros ${ }^{4-7}$. Avanços na técnica cirúrgica e melhor compreensão da anatomia e fisiologia do fígado têm contribuido para diminuição das taxas de insuficiência hepá- tica pós-operatória, reduzindo a morbimortalidade associada às ressecçóes hepáticas. No entanto, estas melhorias não foram acompanhadas por uma reduçáo na taxa de fístula biliar no pós-operatório, que permanece sendo um dos grandes desafios da ressecção hepática ${ }^{5,714-18}$. A incidência de vazamento de bile não mudou ao longo das últimas décadas, variando de $4,0 \%$ a $15 \%$ em estudos recentes ${ }^{19-25}$. A presença da bile na cavidade peritoneal predispóe ao desenvolvimento de sepse, insuficiência hepática e aumenta a mortalidade ${ }^{20,21}$. Assim sendo, muitos métodos foram introduzidos para prevenir extravasamento de bile após a transecção hepática, incluindo colangiografia ${ }^{26}$, aplicação de cola de fibrina na superfície hepática transeccionada ${ }^{27}$ e o teste de vazamento de bile (biliostasia) ${ }^{28}$. Este último é uma abordagem para reduzir a incidência de fístula biliar no pós-operatório ${ }^{29}$. O teste de vazamento de bile provou ser útil na prevençáo de fistula biliar em muitos estudos, entretanto alguns estudos sugerem náo haver vantagem na utilização deste teste, além de que o uso deste teste poderia aumentar o risco de complicaçôes no pós operatório ${ }^{28}$.

Neste contexto, o presente estudo tem como objetivo avaliar se o uso sistemático do teste de biliostasia durante ressecçóes hepáticas reduz a incidência de fístula biliar no pós operatório de ressecçóes hepáticas.

\section{Materiais e MéTOdos}


No período de janeiro 2016 a dezembro 2018 no Hospital Federal de Ipanema foram realizadas 103 hepatectomias. O estudo analisou retrospectivamente pacientes submetidos a hepatectomias realizadas pelo mesmo cirurgiáo. Foram incluídos no estudo 85 pacientes submetidos a hepatectomias maiores ou menores, por doença benigna e maligna. Foram excluídos do estudo 19 pacientes submetidos a cirurgia videolaparoscópica, pacientes submetidos a derivação biliodigestiva e pacientes com dados incompletos. Os pacientes foram divididos em dois grupos: grupo A - pacientes em que náo foi realizado o teste da biliostasia no intra operatório e grupo B - pacientes em que foi realizado o teste da biliostasia no intra operatório. Hepatectomia menor foi definida como ressecção de até 3 segmentos hepáticos e hepatectomia maior como ressecção de 4 ou mais segmentos hepáticos ${ }^{30}$.

O teste da biliostasia foi realizado ao final da transecção hepática. Após realizar colecistectomia, o ducto cístico é cateterizado com um cateter de colangiografia. É injetado através do cateter de colangiografia $10 \mathrm{ml}$ de soro fisiologico $0,9 \%$, seguido de $10 \mathrm{ml}$ de ar duas vezes, observando se há pontos de vazamento de bile na superfície cruenta do fígado. Esses pontos de vazamento são identificados e suturados e o teste é repetido por mais duas vezes. Todos os pacientes submetidos a hepatectomias, incluindo os pacientes em que o teste de biliostasia não foi realizado um dreno tubular aspirativo foi posicionado contemplando a superfície cruenta do fígado.

Fístula biliar após hepatectomia foi definida de acordo com os critérios propostos pelo Grupo Internacional de Estudo em Cirurgia Hepática (ISGLS), assim como o grau de serveridade da fístula ${ }^{31}$. Dessa forma, fístula biliar é definida como fluido com concentraçáo aumentada de bilirrubina no dreno abdominal ou no líquido intraabdominal no $3^{\circ}$ dia pós-operatório ou após a necessidade de drenagem por radiologia intervencionista por biloma ou re-laparotomia por coleperitoneo. $\mathrm{O}$ aumento $\mathrm{da}$ concentração de bilirrubina no dreno ou líquido intra-abdominal é definido como uma bilirrubina pelo menos 3 vezes maior que a concentração sérica de bilirrubina medida no mesmo tempo. Fístula grau A é aquela que requer pouca ou nenhuma alteração no tratamento clínico dos pacientes; fístula grau B é aquela que exige uma mudança no manejo clínico do paciente (por exemplo, diagnóstico adicional ou procedimentos por radiologia intervencionista), mas gerenciáveis sem re-laparotomia, ou uma fístula biliar grau A com duração de mais de 1 semana; fístula grau $C$ é aquela que requer re-laparotomia.

\section{Resultados}


Dos 84 pacientes analisados, 46 $(54,76 \%)$ eram mulheres e $38(45,24 \%)$ homens. Os pacientes foram divididos em dois grupos: Grupo A - pacientes submetidos a ressecção hepática sem teste da biliostasia; Grupo B - pacientes submetidos a ressecção hepática com teste da biliostasia. Quarenta pacientes (47,61\%) foram submetidos a hepatectomia sem teste da biliostasia e alocados no grupo A e 44 pacientes foram submetidos a hepatectomia com teste da biliostasia $(52,39 \%)$ e alocados no grupo B.

Dos pacientes do grupo A, 8 (20\%) foram submetidos a ressecção hepática por doença benigna ( 5 por adenoma e 3 por litíase intrahepática). Trinta e dois pacientes (80\%) foram submetidos a ressecção hepática por doença maligna (17 por metástase hepática de câncer colorretal, 5 por metástase hepática não-colorretal, 5 por neoplasia de vesicular biliar, 2 por carcinoma hepatocelular e 3 por colangiocarcinoma intrahepático). Treze pacientes foram submetidos a hepatectomia maior e 27 submetidos a hepatectomia menor.

Desses pacientes, 5 (12,5\%) evoluíram com fístula biliar no pós operatório. Dois pacientes foram submetidos a ressecção hepática por litíase intrahepática, 1 por carcinoma hepatocelular, 1 por neoplasia de vesícula biliar e 1 por metástase hepática de câncer colorretal. Três pacientes foram submetidos a hepatectomia menor e 2 submetidos a he- patectomia maior. Dessas fístulas, uma foi classificada como grau A, 3 foram classificadas como grau B, necessitando de drenagem por radiologia intervencionista e 1 foi classificada como grau $\mathrm{C}$, necessitando de relaparotomia.

Dos pacientes do grupo B, 4 (9\%) foram submetidos a ressecção hepática por doença benigna ( 2 por adenoma, 1 por hemangioma e 1 por cisto hepático complexo). Quarenta (91\%) pacientes foram submetidos a ressecçáo hepática por doença maligna (28 por metástase hepática de câncer colorretal, 1 por carcinoma hepatocelular, 2 por colangiocarcinoma intrahepático, 6 por neoplasia de vesícula biliar e 3 por metástase hepática não-colorretal). Dezoito pacientes foram submetidos a hepatectomia maior $\mathrm{e}$ 26 submetidos a hepatectomia menor.

Desses pacientes, 2 (4,54\%) evoluíram com fístula biliar no pós operatório. O primeiro paciente, foi submetido a hepatectomia esquerda por adenoma hepático e o teste da biliostasia não evidenciou vazamento e evoluiu com fístula grau B - manejada conservadoramente porém com duração maior que uma semana. O segundo paciente foi submetido a hepatectomia esquerda por metástase hepática de câncer colorretal e o teste da biliostasia evidenciou um ponto de vazamento. Esse paciente também evoluiu com fístula grau B, manejada conservadoramente porém com duração maior que uma semana. 


\section{Discussáo}

A fístula biliar é uma complicação comum após ressecçóes hepáticas e afeta a qualidade de vida no pós operatório e também pode causar infecção intra abdominal e insuficiência hepática 32 . Durante a cirurgia, é difícil para o cirurgião identificar pontos de vazamento de bile e o objetivo do teste de biliostasia é identificar cotos insuficientemente fechados de ductos biliares na superfície transeccionada do fígado e, em seguida, o local de vazamento é suturado.

Durante a última década, vários testes de vazamento de bile foram proposto sem que nenhum deles tenha ganhado ampla aceitação. $\mathrm{O}$ uso apenas de solução salina é uma técnica de baixo custo e reprodutível, porém pode não identificar vazamento em pequenos ductos biliares ${ }^{33}$. $\mathrm{O}$ uso de azul de metileno no teste da biliostasia tem como desvantagem o fato de que a coloração da superfície de transecçáo reduz a sensibilidade para a detecção de vazamento com testes repetidos ${ }^{34}$.

A colangiografia é o teste padrão-ouro para detecção intraoperatória de ductos biliares abertos, mas é demorado e expóe os pacientes e equipe médica à radiação. $\mathrm{O}$ "teste branco", baseado em injeção intraductal de emulsão gordurosa a $5 \%$, foi relatado ser viável, sensível, de baixo custo e reprodutível ${ }^{35}$. Entretanto, a emulsão gor- durosa é um corpo estranho e sua injeção intraductal pode ser associada a efeitos adversos, tais como reações alérgicas, êmbolos gordurosos, efeitos imunossupressores e infecçôes fúngicas.

A única técnica suportada por um ensaio clínico randomizado baseia-se na injeção transductal de verde de indocianina e em seguida realizar uma colangiografia fluorescente usando uma câmera de infravermelho ${ }^{36}$. Este teste aumenta o tempo cirúrgico, requer equipamentos especializados e utiliza algoritmos complexos que indiretamente identificam ductos biliares abertos em um monitor ao executar o teste com a luz da sala de cirurgia apagada. Combinados, cada uma desses métodos de realização do teste de biliostasia coloca obstáculos ao uso generalizado em todas as instituiçôes. Sendo assim, o teste proposto no presente estudo utilizando injeçáo de soro fisiológico 0,9\% seguido de ar através do coto do ducto cístico, é barato, não requer tecnologia adicional, permite repetir o teste conforme necessário e não expõe os pacientes aos riscos associados a outras técnicas.

Alguns estudos ${ }^{20,28,32,37}$ demonstraram que o teste da biliostasia reduz a incidência de fístula biliar no pós operatório, enquanto dois estudos ${ }^{29,34}$ mostraram que não há diferença significativa. $\mathrm{O}$ presente estudo mostrou que o grupo de pacientes submetidos ao teste de biliostasia tiveram menor incidência de fístula biliar no pós operatório 
$(4,45 \%)$ em relação ao grupo de pacientes que não foram submetidos ao teste de biliostasia $(12,5 \%)$. Isto sugere que o teste da biliostasia é um método seguro e eficaz na prevenção da fístula biliar no pós operatório.

Entretanto, no presente estudo, um dos pacientes que evoluiu com fístula biliar no pós operatório, não houve vazamento $\mathrm{du}$ rante o teste da biliostasia. Algumas razóes podem explicar este acontecimento: a pressão no ducto biliar pode não ter sido alta o suficiente para que vazamento de bile fosse observado; a sutura dos pontos de vazamento pode não ter sido feita de forma adequada;parte dos ductos biliares com vazamento não estavam em comunicação com a árvore biliar principal, portanto, os locais de vazamento poderiam não ser identificado através da utilização do teste de biliostasia.

Por outro lado, a maioria dos pacientes que não foram submetidos ao teste de biliostasia não evoluíram com fístula biliar no pós operatório por algumas razóes. Primeiro, os pontos de vazamento menores podem ser fechados espontaneamente.

Segundo, o vazamento de bile de pequenos cotos biliares com alguma comunicação com a árvore biliar principal geralmente fecham espontaneamente com a restauração do peristaltismo e da função da papila duodenal ${ }^{33}$.

\section{Conclusão}

1. Imamura H, Seyama Y, Kokudo N, Maema A, Sugawara Y, Sano K, et al. One thousand fifty-six hepatectomies without mortality in 8 years. Arch Surg. 2003;138:1198-206.

2. Makuuchi M, Sano K. The surgical approach to HCC: our progress and results in Japan. Liver Transpl. 2004;10:S46-52. 
3. Takayama T. Surgical treatment for hepatocellular carcinoma. Jpn J Clin Oncol. 2011;41:447-54.

4. Nordlinger B, Guiguet M, Vaillant JC, et al. Surgical resection of colorectal carcinoma metastases to the liver. A prognostic scoring system to improve case selection, based on 1568 patients. Association Francaise de Chirurgie. Cancer 1996; 77:1254e1262.

5. Jarnagin WR, Gonen M, Fong Y, et al. Improvement in perioperative outcome after hepatic resection: analysis of 1,803 consecutive cases over the past decade. Ann Surg 2002;236: 397 e406.

6. Poon RT, Fan ST. Is primary resection and salvage transplantation for hepatocellular carcinoma a reasonable strategy? Ann Surg 2004;240:925e928; author reply 928 .

7. Belghiti J, Hiramatsu K, Benoist $S$, et al. Seven hundred forty seven hepatectomies in the 1990s: an update to evaluate the actual risk of liver resection. J Am Coll Surg 2000;191:38e46

8. Nordlinger B, Sorbye H, Glimelius $\mathrm{B}$, et al. Perioperative chemotherapy with FOLFOX4 and surgery versus surgery alone for resectable liver metastases from colorectal cancer (EORTC Intergroup trial 40983): a randomised controlled trial. Lancet 2008;371:1007e1016
9. Adam R, Wicherts DA, de Haas RJ, et al. Patients with initially unresectable colorectal liver metastases: is there a possibility of cure? J Clin Oncol 2009;27:1829e1835

10. Ribero D, Abdalla EK, Madoff DC, et al. Portal vein embolization before major hepatectomy and its effects on regeneration, resectability and outcome. Br J Surg 2007;94:1386e1394.

11. Kishi Y, Madoff DC, Abdalla EK, et al. Is embolization of segment 4 portal veins before extended right hepatectomy justified? Surgery 2008;144:744e751

12. Kishi Y, Abdalla EK, Chun YS, et al. Three hundred and one consecutive extended right hepatectomies: evaluation of outcome based on systematic liver volumetry. Ann Surg 2009;250:540e548.

13. Andreou A, Aloia TA, Brouquet A, et al. Recent advances in the curative treatment of colorectal liver metastases. Gastrointest Cancer Res 2011;4[Suppl 1]:S2eS8.

14. Capussotti L, Ferrero A, Vigano $\mathrm{L}$, et al. Bile leakage and liver resection: where is the risk? Arch Surg 2006;141:690e694; discussion 695.

15. Cescon M, Vetrone G, Grazi GL, et al. Trends in perioperative outcome after hepatic resection: analysis of 1500 
consecutive unselected cases over 20 years. Ann Surg 2009;249:995e1002.

16. Lo CM, Fan ST, Liu CL, et al. Biliary complications after hepatic resection: risk factors, management, and outcome. Arch Surg 1998;133:156e161.

17. Imamura $H$, Seyama $Y$, Kokudo $\mathrm{N}$, et al. One thousand fifty six hepatectomies without mortality in 8 years. Arch Surg 2003;138:1198e1206.

18. Zimmitti G, Roses RE, Andreou A, et al. Greater complexity of liver surgery is not associated with an increased incidence of liver-related complications except for bile leak: an experience with 2,628 consecutive resections. J Gastrointest Surg 2013; 17:57e64; discussion 6465

19. Capussotti L, Ferrero A, Viganò L, Sgotto E, Muratore A, Polastri R. Bile leakage and liver resection: Where is the risk? Arch Surg 2006; 141: 690-694; discussion 695 [PMID: 16847242 DOI: 10.1001/ archsurg.141.7.690]

20. Lam CM, Lo CM, Liu CL, Fan ST. Biliary complications during liver resection. World J Surg 2001; 25: 1273-1276 [PMID: 11596889 DOI: 10.1007/s00268-001-0109-1]

21. Guillaud A, Pery C, Campillo B, Lourdais A, Sulpice L, Boudjema K. Incidence and predictive factors of clinically relevant bile leakage in the modern era of liver resections. HPB (Oxford) 2013; 15: 224-229 [PMID: 23374363 DOI: $10.1111 /$ j.14772574.2012.00580.x]

22. Lo CM, Fan ST, Liu CL, Lai EC, Wong J. Biliary complications after hepatic resection: risk factors, management, and outcome. Arch Surg 1998; 133: 156-161 [PMID: 9484727]

23. Nagano Y, Togo S, Tanaka K, Masui H, Endo I, Sekido H, Nagahori $\mathrm{K}$, Shimada H. Risk factors and management of bile leakage after hepatic resection. World J Surg 2003; 27: 695-698 [PMID: 12732991 DOI: 10.1007/s00268-003-6907-x]

24. Tanaka S, Hirohashi K, Tanaka H, Shuto T, Lee SH, Kubo S, Takemura S, Yamamoto T, Uenishi T, Kinoshita $H$. Incidence and management of bile leakage after hepatic resection for malignant hepatic tumors. J Am Coll Surg 2002; 195: 484-489 [PMID: 12375753 DOI: $10.1016 / S 1072-$ 7515(02)01288-7]

25. Yamashita Y, Hamatsu T, Rikimaru T, Tanaka S, Shirabe K, Shimada M, Sugimachi K. Bile leakage after hepatic resection. Ann Surg 2001; 233: 4550 [PMID: 11141224 DOI: 10.109 7/00000658-200101000-00008]

26. Kubo S, Sakai K, Kinoshita H, Hirohashi K. Intraoperative 
cholangiography using a balloon catheter in liver surgery. World J Surg 1986; 10: 844-850 [PMID: 3776221

DOI: 10.1007/ BF01655256]

27. Figueras J, Llado L, Miro M, Ramos E, Torras J, Fabregat J, Serrano T. Application of fibrin glue sealant after hepatectomy does not seem justified: results of a randomized study in 300 patients. Ann Surg 2007; 245: 536-542 [PMID: 17414601DOI: 10.1097/01. sla.0000245846.37046.57]

28. Wang HQ, Yang J, Yang JY, Yan LN. Bile Leakage test in liver resection: A systematic review and meta-analysis. World J Gastroenterol 2013; 19(45): 8420-8426 Available from: URL: http://www.wignet.com/1007-9327/ full/v19/i45/8420.htm DOI: http:// dx.doi.org/10.3748/wjg.v19.i45.8420

29. Ijichi M, Takayama T, Toyoda $\mathrm{H}$, Sano K, Kubota K, Makuuchi M. Randomized trial of the usefulness of a bile leakage test during hepatic resection. Arch Surg 2000; 135: 1395-1400 [PMID: 11115338 DOI: 10.1001/archsurg.135.12.1395

30. Reddy SK, Barbas AS, Turley RS, et al. A standard definition of major hepatectomy: resection of four or more liver segments. HPB (Oxford) 2011;13:494-502. 10.1111/j.14772574.2011.00330.x
31. Koch M, Garden OJ, Padbury R, Rahbari NN, Adam R, Capussotti L, et al. Bile leakage after hepatobiliary and pancreatic surgery: a definition and grading of severity by the International Study Group of Liver Surgery. Surgery. 2011; 149:680-8.

32. Liu Z, Jin H, Li Y, Gu Y, Zhai C. Randomized controlled trial of the intraoperative bile leakage test in preventing bile leakage after hepatic resection. Dig Surg 2012; 29: 510 515 [PMID: 23392477 DOI: $10.1159 / 000346480]$

33. Ijichi M, Takayama T, Toyoda $\mathrm{H}$, et al. Randomized trial of the usefulness of a bile leakage test during hepatic resection. Arch Surg 2000;135:1395e1400.

34. Suehiro T, Shimada M, Kishikawa K, et al. In situ dye injection bile leakage test of the graft in living donor liver transplantation. Transplantation 2005;80:1398e1401.

35. Nadalin S, Li J, Lang $\mathrm{H}$, et al. The white test: a new dye test for intraoperative detection of bile leakage during major liver resection. Arch Surg 2008;143:402e404; discussion 404

36. Kaibori M, Ishizaki M, Matsui K, et al. Intraoperative indocyanine green fluorescent imaging for prevention of bile leakage after hepatic resection. Surgery 2011;150:91e98. 
37. Li J, Malagó M, Sotiropoulos GC, Lang H, Schaffer R, Paul A, Broelsch CE, Nadalin S. Intraoperative application of "white test" to reduce postoperative bile leak after major liver resection: results of a prospective cohort study in 137 patients.

Langenbecks Arch Surg 2009; 394:

1019-1024 [PMID: 19104825] 